\title{
Temporal Spectroscopic Characteristics of Terahertz Pulses Transmitted through Metal Hole Arrays
}

\author{
Fumiaki Miyamaru Non-member \\ Masanori Hangyo Non-member
}

Keywords : metal hole array, extraordinary transmission, $\mathrm{THz}$ time domain spectroscopy

Since Ebbesen et al. reported the extraordinary transmission property for the metal hole array in 1998, many studies have been carried out both experimentally and theoretically to understand the physical mechanism of this phenomenon. Until now, it is widely supported that the extraordinary optical transmission is attributed to the resonant excitation of the surface plasmon-polaritons (SPPs) by the incident light on the periodically modulated metal-air (or dielectric) boundary. Although large amount of papers have been reported, most of them are focused on the stationary transmission spectrum of periodical metal hole arrays and metallic slit gratings. Not only the stationary transmittance for the metal hole arrays but also the temporal behavior of light are very important for applying the metal hole arrays to ultrafast optical devices. In this paper, we measured the temporal transmission property of the $\mathrm{THz}$ pulse in order to investigate the time-dependent transmission characteristics of the metal hole array.

We measured the THz wave form transmitted through the metal hole arrays, which are aluminum slabs perforated with circular holes in the triangular structure (Fig. 1), by using the terahertz time domain spectroscopy (THz-TDS). The temporal wave form of the $\mathrm{THz}$ wave transmitted through the metal hole array at normal incidence is shown in Fig. 2(b). The incident THz wave is also shown in Fig 2(a) as reference. While the incident $\mathrm{THz}$ wave is an almost single cycle pulse, the transmitted $\mathrm{THz}$ wave has a sharp peak at around $t=0 \mathrm{ps}$ followed by over ten cycle oscillations. This oscillation component corresponds to the $\mathrm{THz}$ wave reemitted from the SPPs which are excited on both surfaces of the metal hole array by the incident $\mathrm{THz}$ wave through the resonant interaction mediated by the periodic corrugation of the metal surface. The decay time of this oscillation corresponds to the life time of the SPP, which is estimated to be $\tau=17$ ps by the numerical fitting.

The temporal spectroscopic behavior of the transmitted $\mathrm{THz}$ wave can also be analyed from the temporal waveform. In order to obtain the time-dependent spectroscopic behavior of the transmitted $\mathrm{THz}$ wave, the Gabor transformation was performed with the variable Gaussian time window. Result is shown in Fig.2

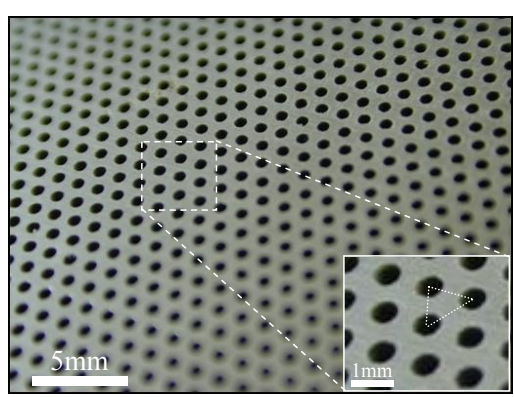

Fig. 1. Photograph of the metal hole array (c). In the frequency region higher than $0.30 \mathrm{THz}$, the $\mathrm{THz}$ wave has very short rise and decay times, and has the maximum amplitude at around $t=0 \mathrm{ps}$. At the resonant frequency of the SPP $(0.27 \mathrm{THz})$, on the other hand, the transmission intensity rises relatively slowly and becomes maximum at around $t=10 \mathrm{ps}$, indicating that the $\mathrm{THz}$ wave at the resonant frequency of the SPP propagates more slowly than the $\mathrm{THz}$ wave directly transmitted through the metal holes at higher frequencies. This caharacteristic is dominated by the deceleration of the group velocity of the $\mathrm{THz}$ wave in the metal hole.

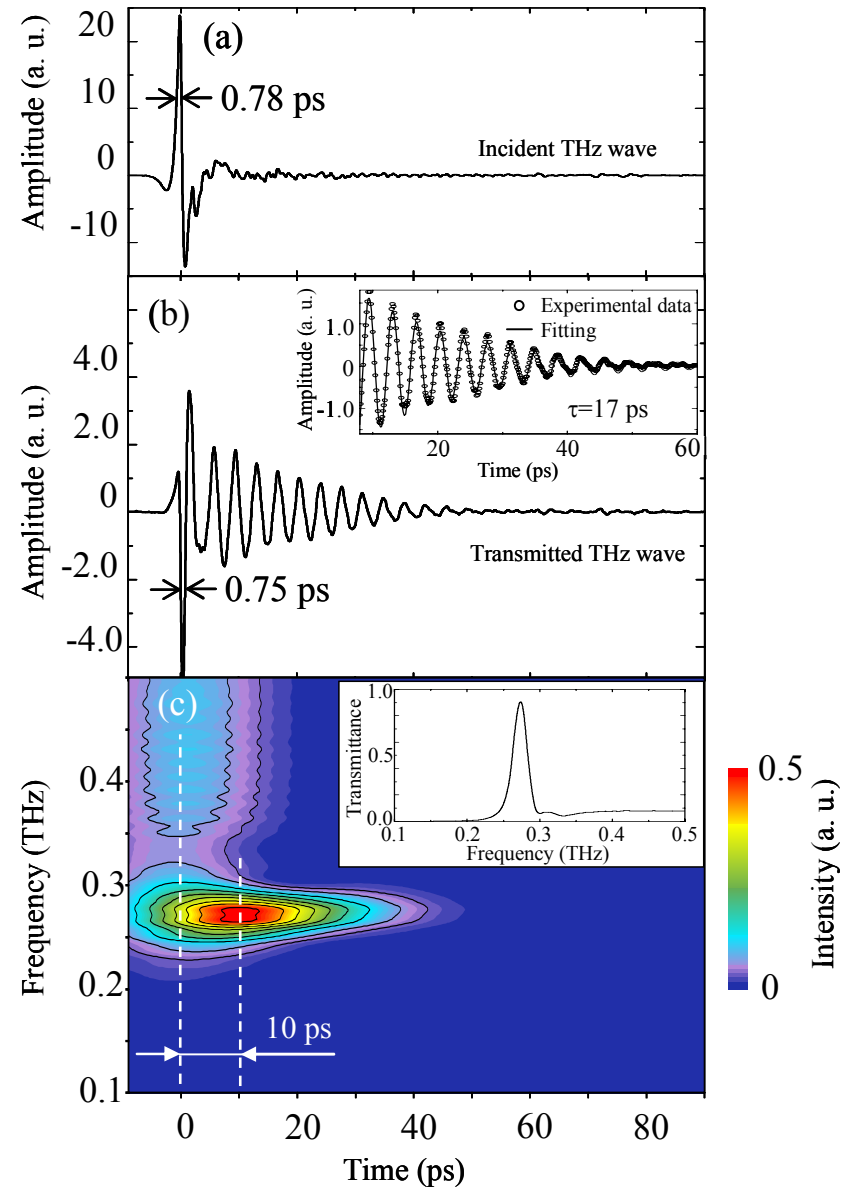

Fig. 2. (a) Measured waveform of the incident $\mathrm{THz}$ wave, (b) Measured waveform of the $\mathrm{THz}$ wave transmitted through the metal hole array; Inset shows the expanded waveform (open circle) in the range from 10 to $60 \mathrm{ps;}$ The numerical fiited curve with a damped harmonic oscillator is also shown (solid line), (c) Gabor spectrum obtained with the variable Gaussian time window of the window width $\delta=34 \mathrm{ps}$; Inset shows the transmission spectrum of the metal hole array 


\title{
Temporal Spectroscopic Characteristics of Terahertz Pulses Transmitted through Metal Hole Arrays
}

\author{
Fumiaki Miyamaru* Non-member \\ Masanori Hangyo ** Non-member
}

The temporal spectroscopic characteristics of the terahertz pulse transmitted through the metal hole array is investigated by using the THz time domain spectroscopy in combination with the short-time Fourier transformation. It is found that the $\mathrm{THz}$ wave at around the resonant frequency of the surface plasmon-polariton (SPP) delays compared with that at higher frequency region. From the dependence of the delay time on the thickness of the metal hole array and the frequency, the delay at the SPP resonant frequency is attributed to the deceleration of the group velocity of the THz wave in the metal hole.

Keywords : metal hole array, extraordinary transmission, $\mathrm{THz}$ time domain spectroscopy

\section{Introduction}

Since Ebbesen et al. reported the extraordinary transmission property for the metal hole array in $1998^{(1)}$, many studies have been carried out both experimentally and theoretically to understand the physical mechanism of this phenomenon ${ }^{(2)-(5)}$. Until now, it is widely supported that the extraordinary optical transmission is attributed to the resonant excitation of the surface plasmon-polaritons $(\mathrm{SPPs})^{(6)}$ by the incident light on the periodically modulated metal-air (or dielectric) boundary. Although large amount of papers have been reported, most of them are focused on the stationary transmission spectrum of periodical metal hole arrays and metallic slit gratings. Only a few papers deal with the optical pulse propagation through metallic periodic structures ${ }^{(7)(8)}$. Not only the stationary transmittance for the metal hole arrays but also the temporal behavior of light are very important for applying the metal hole arrays to ultrafast optical devices. In Ref. (7), the time delay of the optical pulse propagation through the metal aperture array has been observed, and such time delay is attributed to the resonant coupling of the incident light with the surface modes excited on the metal surface. However, the low group velocity of the optical pulse should be also included as the additional mechanism for the observed time delay in the experiment of Ref. (7). In this paper, we measured the temporal transmission property of the terahertz $(\mathrm{THz})$ pulse in order to investigate the time-dependent transmission characteristics of the metal hole array, and found the significant contribution of the delay owing to the low group velocity of the $\mathrm{THz}$ pulse in the metal holes.

The anomalously high band-pass transmission property for the metal hole arrays has been reported experimentally in the millimeter and $\mathrm{THz}$ regions ${ }^{(9)-(14)}$. In most of the experiments in these regions, the band-pass peak frequency is located above the cutoff frequency of circular metal holes, indicating that the electromagnetic wave is propagation mode in the metal hole. This situation is different from that of the sub-wavelength metal hole

\footnotetext{
* Faculty of Scince, Shinshu University Asahi 3-1-1, Matsumoto, Nagano 390-8621

** Institute of Laser Engineering, Osaka University

Yamadaoka 2-6, Suita, Osaka 565-0871
}

arrays in the visible region ${ }^{(1)}$ where the electromagnetic wave is the evanescent mode in the metal hole. Despite of such the difference, the mechanism of the high band-pass transmission property in the $\mathrm{THz}$ region is analogous to that of the Ebbesens' experiment in the visible region ${ }^{(2)(11)(15)}$.

\section{Experient}

We measured the $\mathrm{THz}$ wave form transmitted through the several types of a metal hole array, which are aluminum slabs perforated with circular holes in the triangular structure (Fig. 1), by using the $\mathrm{THz}$ time domain spectroscopy (THz-TDS). The fundamental measurement principle of this system is as follows. The $\mathrm{THz}$ wave was generated by illuminating a photoconductive antenna evaporated on a low-temperature-grown gallium arsenide (LT-GaAs) substrate with $100 \mathrm{fs}$ pump laser pulses. The THz wave was collimated by an off-axis paraboloidal mirror and impinged on the sample. After transmitted through the sample, the $\mathrm{THz}$ wave was focused on another photoconductive antenna which was illuminated by gate laser pulses separated from the pump laser pulses. By changing the delay between the pump and gate laser pulses, the temporal behavior of the electric field of the $\mathrm{THz}$ wave were measured. The transmission spectrum was obtained by dividing the Fourier component of the transmitted $\mathrm{THz}$ wave by that of a reference $\mathrm{THz}$ wave.

\section{Results and Discussion}

The temporal waveform of the $\mathrm{THz}$ wave transmitted through the metal hole array at normal incidence is shown in Fig. 2(b). The

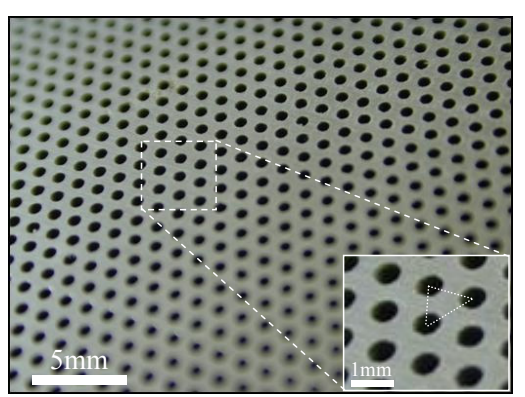

Fig. 1. Photograph of the metal hole array 
incident $\mathrm{THz}$ wave is also shown in Fig 2(a) as reference. The geometrical parameters of the metal hole array are as follows: a diameter $d=0.68 \mathrm{~mm}$, a lattice constant $s=1.13 \mathrm{~mm}$, and a slab thickness $l=0.50 \mathrm{~mm}$. While the incident $\mathrm{THz}$ wave is an almost single cycle pulse, the transmitted $\mathrm{THz}$ wave has a sharp peak at around $t=0 \mathrm{ps}$ followed by over ten cycle oscillations. The first sharp peak at around $t=0 \mathrm{ps}$ corresponds to the $\mathrm{THz}$ wave transmitted through the metal hole directly without the resonant coupling to the SPPs. Below the cutoff frequency $\left(f_{\mathrm{c}}=0.26 \mathrm{THz}\right)$, an electromagnetic wave becomes an evanescent wave in the metal hole, and therefore, the transmittance becomes extremely low ${ }^{(16)}$. Such a high-pass filtering effect makes the pulse width of the transmitted $\mathrm{THz}$ wave at $\mathrm{t} \sim 0 \mathrm{ps}$ shorter than that of the incident $\mathrm{THz}$ wave as seen in Fig. 2(a). The oscillation following the sharp pulse at around 0 ps corresponds to the $\mathrm{THz}$ wave reemitted from the $\operatorname{SPPs}^{(17)}$ which are excited on both surfaces of the metal hole array by the incident $\mathrm{THz}$ wave through the resonant interaction mediated by the periodic corrugation of the metal surface. The decay time of this oscillation corresponds to the lifetime of the SPP. In order to estimate the lifetime of the SPP, we performed the numerical fitting to the transmitted $\mathrm{THz}$ waveform with the damped harmonic oscillation in the time range after $t=8 \mathrm{ps}$. As shown in the inset of Fig. 2(b), the oscillation component can be fitted well with the damped harmonic oscillator, and the decay time $\tau$ is estimated to be $\tau=17$ ps which is 4.6 times as long as the oscillation period.

Next, we investigate the temporal spectroscopic behavior of the transmitted $\mathrm{THz}$ wave. The transmission spectrum of the metal hole array is shown in the inset of Fig. 2(c). The transmission peak is observed at $0.27 \mathrm{THz}$ which corresponds to the oscillation component observed in the transmitted $\mathrm{THz}$ wave form, and below $0.25 \mathrm{THz}$ the transmittance is almost zero because of the cutoff effect of the metal hole. In the frequency region above $0.30 \mathrm{THz}$, the $\mathrm{THz}$ wave transmits directly through the metal hole. In this region, the transmittance is expected to be same with the air fraction $F_{\text {air }}$ of metal holes given by

$$
F_{\text {air }}=\frac{\pi}{2 \sqrt{3}}\left(\frac{d}{s}\right)^{2}
$$

However, the transmittance is at most $10 \%$ which is considerably smaller than the expected value of our metal hole arrays $\left(F_{\text {air }} \sim\right.$ $0.33)$. This discrepancy is due to the diffraction loss to the higher diffraction orders ${ }^{(9)}$, because in our experimental setup only the zeroth order transmission component can be detected. In order to obtain the time-dependent spectroscopic behavior of the transmitted $\mathrm{THz}$ wave, the Gabor transformation is performed with the variable Gaussian time window. By changing the delay time of the Gaussian time window, the time evolution of the transmittance (Gabor spectrum) can be obtained as shown in Fig. 2(c). In this analysis, the window width $\delta$ of the Gaussian time window determines both the time and frequency resolutions of the Gabor spectrum. The time resolution increases proportionally with decreasing $\delta$, while the frequency resolution decreases with decreasing $\delta$. In order to obtain the appropriate time and frequency resolutions, we select the window width to be $\delta=34$ ps in this analysis. At $0.50 \mathrm{THz}$, the $\mathrm{THz}$ wave has very short rise and decay times, and has the maximum amplitude at around $t=0 \mathrm{ps}$. At the resonant frequency of the SPP $(0.27 \mathrm{THz})$, on the other hand, the transmission intensity rises relatively slowly and becomes maximum at around $t=10 \mathrm{ps}$, indicating that the $\mathrm{THz}$ wave at the

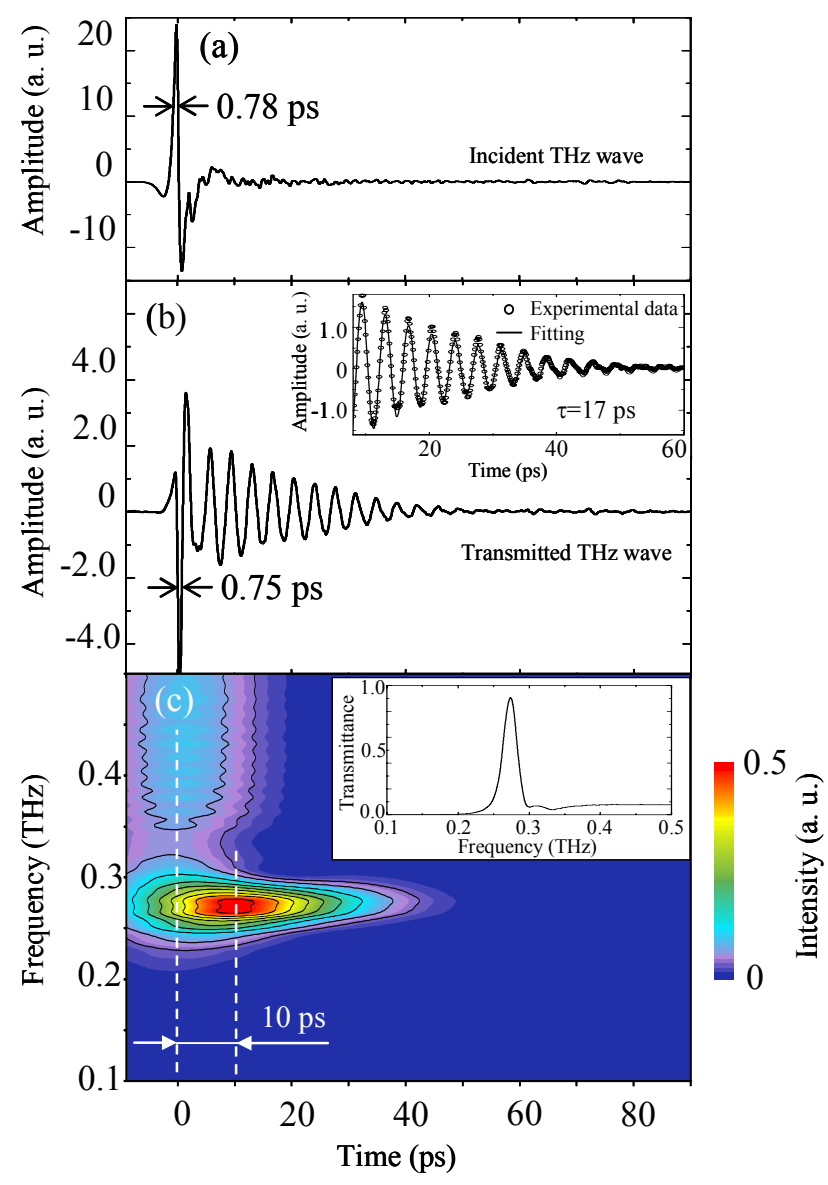

Fig. 2. (a) Measured waveform of the incident $\mathrm{THz}$ wave, (b) Measured waveform of the $\mathrm{THz}$ wave transmitted through the metal hole array; Inset shows the expanded waveform (open circle) in the range from 10 to $60 \mathrm{ps}$; The numerical fiited curve with a damped harmonic oscillator is also shown (solid line), (c) Gabor spectrum obtained with the variable Gaussian time window of the window width $\delta=34 \mathrm{ps}$; Inset shows the transmission spectrum of the metal hole array

resonant frequency of the SPP propagates more slowly than the $\mathrm{THz}$ wave directly transmitted through the metal holes at higher frequencies.

Two mechanisms can be considered as the reason for the propagation delay of the $\mathrm{THz}$ wave at $0.27 \mathrm{THz}$. One is the delay due to the conversion time from the incident $\mathrm{THz}$ wave to the SPP at the input surface and from the SPP to the outgoing $\mathrm{THz}$ wave at the output surface. Another possible mechanism is the deceleration of the group velocity of the $\mathrm{THz}$ wave when the $\mathrm{THz}$ wave propagates in the metal holes ${ }^{(16)}$. In order to investigate which mechanism is dominant for the delay time observed in Fig. 2(c), we measured the dependence of the delay time on the thickness of the metal hole array. If the former mechanism is dominant, the delay time should be almost independent of the thickness because the conversion time of the SPP is considered to be sensitive only to the structure of the metal surface. If the latter mechanism, on the other hand, is dominant, the increase of the delay time should be observed with increasing the thickness of the metal hole array. Figures 3(a)-(d) show the Gabor spectra of the $\mathrm{THz}$ waves transmitted through the metal hole arrays for the thicknesses of $l=0.20,0.50,1.00$ and $2.00 \mathrm{~mm}$, respectively. The hole diameter 


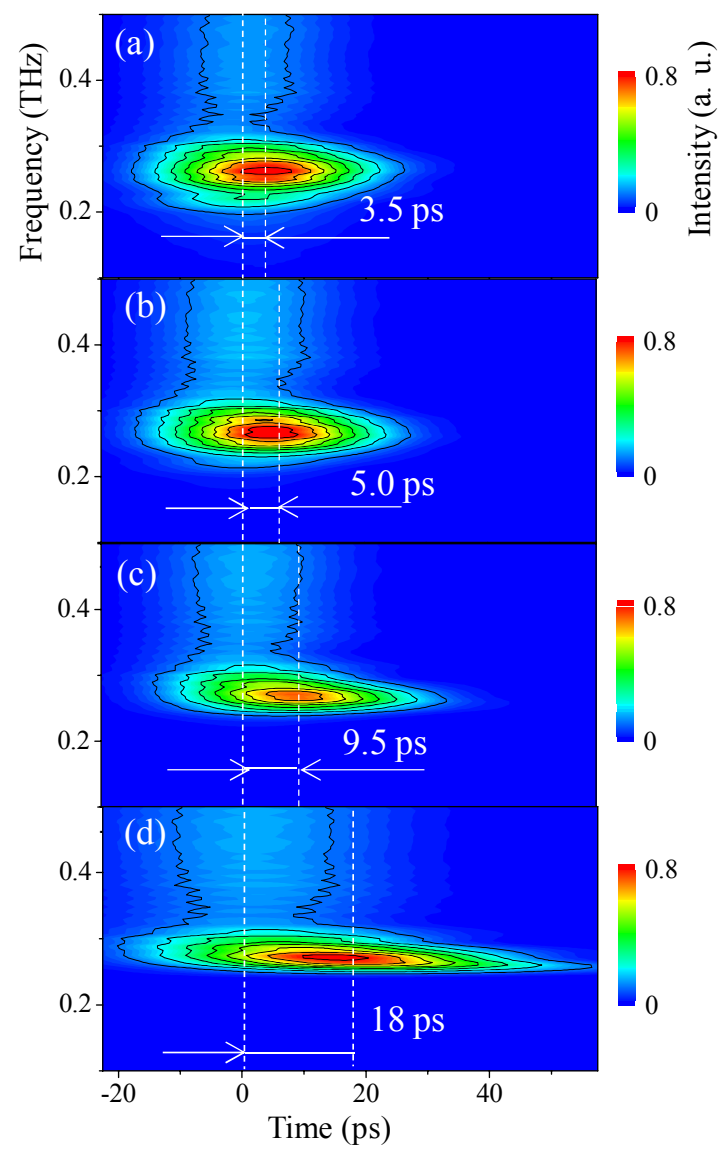

Fig. 3. Gabor spectra for metal hole arrays with several thicknesses of (a) $0.20 \mathrm{~mm}$, (b) $0.50 \mathrm{~mm}$, (c) $1.00 \mathrm{~mm}$ and (d) $2.00 \mathrm{~mm}$

and the lattice constant are $d=0.70$ and $s=1.12 \mathrm{~mm}$, respectively, for all samples. From these figures, it is clearly observed that the delay time between maximum points of the transmission peak frequency $(0.27 \mathrm{THz})$ and the higher frequency region increases systematically from $\Delta t=3.5$ to 18 ps with increasing the thickness from 0.20 to $2.00 \mathrm{~mm}$. This result demonstrates that the dominant contribution to the delay is the deceleration of the $\mathrm{THz}$ wave group velocity at the transmission peak frequency. In the microwave theory ${ }^{(15)}$, the group velocity of the electromagnetic wave in the perfect conductor circular hole is expressed by

$$
v_{g}(f)=c \sqrt{1-\left(f_{c} / f\right)^{2}}
$$

Here, $f_{\mathrm{c}}=1.841 c / \pi d$ is the cutoff frequency of the $\mathrm{TE}_{11}$ mode which is the lowest order of the waveguide mode of the metal hole. $f$ and $c$ are the frequency of the electromagnetic wave and the velocity of light in vacuum, respectively. Figure 4 shows the frequency dependence of the measured (closed triangle) and calculated (open circle) delay times of the $\mathrm{THz}$ wave with respect to that at 0.50 $\mathrm{THz}$ for the metal hole array $(d=0.70 \mathrm{~mm}, s=1.12 \mathrm{~mm}, l=2.00$ $\mathrm{mm})$. The delay time $\Delta t$ is calculated from $\Delta t(f)=l\left(1 / v_{\mathrm{g}}(0.50 \mathrm{THz})\right.$ $\left.1 / v_{\mathrm{g}}(f)\right)$. The cutoff frequency $f_{\mathrm{c}}(0.25 \mathrm{THz})$ is shown as a vertical dashed line in the figure. The good agreement between the experiment and theory is observed. This result also indicates that the deceleration of the $\mathrm{THz}$ wave group velocity is the dominant mechanism for the propagation delay of the $\mathrm{THz}$ wave. The discrepancy at around $0.32 \mathrm{THz}$ might be due to the effect of the resonant excitation of the SPP, which induces the negative group

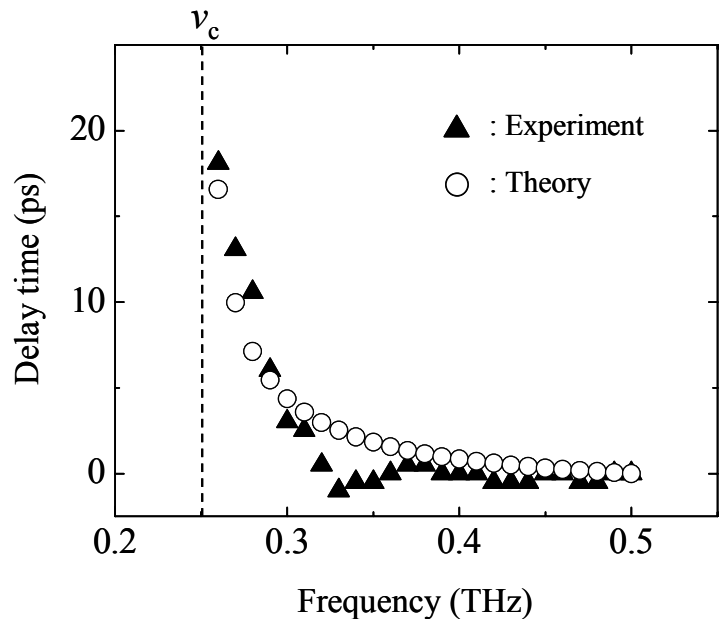

Fig. 4. Measured (triangle) and calculated (circle) delay time of the $\mathrm{THz}$ wave as a function of the frequency with respect to that at $0.50 \mathrm{THz}$ for the metal hole array of $d=0.70 \mathrm{~mm}, s=1.12$ $\mathrm{mm}$, and $l=2.00 \mathrm{~mm}$

delay for the electromagnetic wave at around the resonant frequency. Details about such a negative group velocity delay effect for the $\mathrm{THz}$ wave transmitted through the metal hole array will be discussed elsewhere.

\section{Summary}

In summary, we investigated the temporal spectroscopic behavior of the $\mathrm{THz}$ wave transmitted through the metal hole array by using the Gabor transformation. In the Gabor spectrum, the propagation delay of the $\mathrm{THz}$ wave at the transmission peak frequency compared with that of the higher frequency range is observed. The dominant mechanism of this delay is the deceleration of the group velocity of the $\mathrm{THz}$ wave in the metal hole. The conversion time between the SPPs and free-space $\mathrm{THz}$ waves plays a minor role for the propagation delay time.

This work was partially supported by the Japan Society for Promotion of Science.

(Manuscript received Nov. 21, 2008, revised Feb. 18, 2009)

\section{References}

(1) T. W. Ebbesen, L. J. Lezec, H. F. Ghaemi, T. Thio, and P. A. Wolff : "Extraordinary optical transmission through sub-wavelength hole arrays", Nature, Vol.391, pp.667-669, London (1998)

(2) L. Martín-Moreno, F. J. Garcia-Vidal, H. J. Lezec, K. M. Pellerin, T. Thio, J. B. Pendry, and T. W. Ebbesen : "Theory of Extraordinary Optical Transmission through Subwavelength Hole Arrays", Phys. Rev. Lett., Vol.86, pp.1114-1117 (2001)

(3) D. E. Grupp, H. J. Lezec, T. W. Ebbesen, K. M. Pellerin, and T. Thio : "Crucial role of metal surface in enhanced transmission through subwavelength apertures", Appl. Phys. Lett., Vol.77, pp.1569-1571 (2000)

(4) S. A. Darmanyan and A. V. Zayats : "Light tunneling via resonant surface plasmon polariton states and the enhanced transmission of periodically nanostructured metal films: An analytical study", Phys. Rev. B, Vol.67, p.35424 (2003)

(5) W. L. Barnes, W. A. Murray, J. Dintinger, E. Davaux, and T. W. Ebbesen : "Surface Plasmon Polaritons and Their Role in the Enhanced Transmission of Light through Periodic Arrays of Subwavelength Holes in a Metal Film", Phys. Rev. Lett., Vol.92, p.107401 (2004)

(6) H. Raether, Surface Plasmons on Smooth and Rough Surfaces and on Gratings, Springer-Verlag, Berlin (1988)

(7) A. Dogariu, T. Thio, L. J. Wang, T. W. Ebbesen, and H. J. Lezec : "Delay in light transmission through small apertures", Opt. Lett., Vol.26, pp.450-452 (2001) 
(8) A. Dechant and A. Y. Elezzabi : "Femtosecond optical pulse propagation in subwavelength metallic slits", Appl. Phys. Lett., Vol.84, p.4678 (2004)

(9) C. Winnewisser, F. T. Lewen, M. Schall, M. Walther, and H. Helm : IEEE Trans. Microwave Theory Tech., Vol.48, p.744 (2000)

(10) F. Miyamaru, T. Kondo, T. Nagashima, and M. Hangyo : "Large polarization change in two-dimensional metallic photonic crystals in subterahertz region", Appl. Phys. Lett., Vol.82, pp.2568-2570 (2003)

(11) F. Miyamaru and M. Hangyo : "Finite size effect of transmission property for metal hole arrays in subterahertz region", Appl. Phys. Lett., Vol.84, pp.2742-2744 (2004)

(12) F. Keilmann : "Infrared high-pass filter with high contrast", Int. J. Infrared \& Millimeter Waves, Vol.2, pp.259-272 (1981)

(13) R. Ulrich : "Modes of propagation on an open periodic waveguide for the far infrared", Proc. of the Symposium on Optical and Acoustical Microelectronics, J. Fox, Ed. pp.359-376, Polytechnic Press, Brooklyn, New York (1974)

(14) K. Sakai and L. Genzel : Review of Infrqred and Millimeter Waves, K. J. Button, Ed., Plenum Press, New York (1983)

(15) L. Sarrazin, J.-P. Vigneron, and J.-M. Vigoureux : Phys. Rev. B, Vol.67, 085415 (2003)

(16) G. Torosyan, C. Rau, B. Pradarutti, and R. Beigang : "Generation and propagation of surface plasmons in periodic metallic structures", Appl. Phys. Lett., Vol.85, p.3372 (2004)

(17) N. Marcuvitz : Waveguide Handbook, McGraw-Hill, New York (1951)
Fumiaki Miyamaru

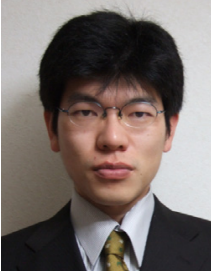

Non-member) received a $\mathrm{Ph}$. D. degree in Engineering from Osaka University in 2004, and is presently an assistant proffecer at Shinshu University. He has worked on photonic crystals, and development of terahertz spectroscopy. Current interest is in metamaterials in terahertz region. Japan Applied Physics Society, Japan Physical Society, American Physical Society member.

Masanori Hangyo

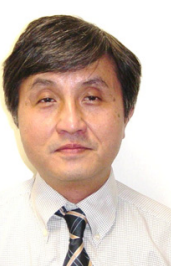

(Non-member) received a $\mathrm{Ph}$. D. degree in Science from Kyoto University in 1981, and is presently a professorr of Institute of Laser Engineering, Osaka University. His interest is in terahertz technology using femtosecond lasers and its applications to various fields. $\mathrm{He}$ is a member of Japanese Society of Applied Physics. 